\title{
El impacto del intercambio de música sobre la compra de discos y la asistencia a conciertos. El caso de España
}

\section{Manuel Herrera-Usagre}

Universidad de Sevilla. Agencia de Calidad Sanitaria de Andalucía

mherrera3@us.es

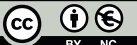

Recibido: 13-09-2011

Aceptado: 29-11-2011

\section{Resumen}

En la última década, el intercambio de archivos a través de Internet ha pasado de ser un fenómeno circunscrito a una minoría de la población, a convertirse en un fenómeno de gran impacto a nivel social, mediático, económico y político. El motivo no es otro que la percepción de un elemento de causalidad directa entre el incremento de las descargas y la disminución de las compras de bienes culturales como los discos de música. Es lo que hemos denominado la perspectiva de la sustitución utilitarista. No obstante, otros estudios afirman que el intercambio a través de redes P2P no sustituye a la compra. Es lo que hemos denominado la perspectiva de la complementariedad del consumo. El presente trabajo pretende observar empíricamente esas relaciones (descarga y compra, y descarga y asistencia a conciertos) en el comportamiento de los individuos, utilizando para ello la Encuesta de hábitos de prácticas culturales (SGAE, Ministerio de Cultura, 2007) y aplicando dos técnicas multivariables: la regresión lineal de mínimos cuadrados (para el número de discos comprados) y la regresión logística (para la asistencia a conciertos). Como resultado, hemos averiguado que cuantos más discos intercambian los individuos, más discos compran en formato físico y más probabilidades tienen de acudir a conciertos. Además, encontramos otras variables que influyen en ambos comportamientos, como el nivel educativo, la edad, la situación socioprofesional o el ciclo vital asociado a la paternidad o la maternidad.

Palabras clave: consumo cultural; industria de la música; Internet; intercambio de archivos; redes P2P; cambio tecnológico.

Abstract. The Impact of Music Sharing on Album Purchases and Concert Attendance: The Case of Spain

In the last decade, digital file sharing has gone from being a phenomenon confined to a small share of the population to become a phenomenon with a great social, media, economic and political impact. The reason is none other than the perception of a direct causal element between increasing downloading practices and decreasing purchases of cultural goods such as music albums, what we call the Replacing Utilitarian Perspective. In contrast, 
other studies argue that downloading practices on P2P networks do not replace the purchase of music albums, what we call the Complementary Consumption Perspective. This paper aims to empirically examine this relationship (download-purchase and download-attending concerts) in individuals' behavior using the Cultural Habits and Practices Survey (SGAE, Spanish Ministry of Culture, 2007) and applying two multivariate techniques: linear regression for the number of albums purchased and logistic regression for attendance to concerts. Our results reveal that the more albums individuals share, the more albums they purchase in physical format, and the more likely they are to attend concerts. In addition, other variables influence both behaviors such as educational level, age, socio-professional situation or life cycle associated with paternity/maternity experience.

Keywords: cultural consumption; music industry; the Internet; file sharing; P2P networks; technological change.
Sumario
1. Introducción
6. Resultados
2. La música como flujo de información
7. Conclusiones
3. Las redes peer-to-peer
8. Discusión
4. Hipótesis
Referencias bibliográficas

5. Métodos y datos

\section{Introducción}

En la última década, el fenómeno del intercambio de archivos a través de Internet ha pasado de ser un fenómeno anecdótico y circunscrito a una minoría de la población, a convertirse en un fenómeno de gran impacto a nivel social, mediático, económico y político. Sin dejar de ser un comportamiento minoritario, su regularización ha sido intensamente demandada por un amplio sector de la cultura, tanto productores y distribuidores, como creadores y trabajadores en general. El motivo no es otro que la percepción de un elemento de causalidad directa entre el incremento de las descargas y la disminución de las compras de bienes culturales como los discos de música. El intercambio digital es concebido por el sector como la primera de las causas que han hecho zozobrar recientemente la industria de la cultura audiovisual. El establecimiento de esta relación causa-efecto se viene realizando a través de numerosos informes que utilizan datos agregados, como el número de descargas digitales realizadas en los últimos años y el número de venta de discos en el mismo periodo. A medida que se han ido observando un aumento de las descargas y una disminución de las ventas, la industria cultural se ha aferrado al argumento de la existencia de una tasa de sustitución de material en venta por material pirata. En otras palabras, cada disco o película que nos descargamos es un disco o película menos que hubiésemos comprado. He aquí la causa, al menos en parte, de las pérdidas de la industria. Con el presente trabajo, pretendemos observar empíricamente 
esa relación (descarga-compra) en el comportamiento de los individuos, utilizando para ello fuentes primarias de comportamiento como son las encuestas. En nuestro caso, utilizaremos la Encuesta de hábitos y prácticas culturales -EHPC a partir de ahora- (Fundación Autor-SGAE y Ministerio de Cultura, 2007) para realizar los siguientes análisis: en primer lugar, queremos establecer qué características sociales influyen en la compra de discos. Comprobaremos si la descarga o la grabación de música supone un receso o un aumento en dichas compras. En segundo lugar, analizaremos también las características sociales que influyen en la asistencia a conciertos de música en vivo. Esta práctica, que, según los datos que maneja la industria, está cada vez más extendida, supone una recompensa económica directa a los creadores. Comprobaremos también si la asistencia está influenciada por una mayor descarga o grabación de discos. Finalmente, presentaremos las conclusiones más relevantes.

\section{La música como flujo de información}

John Perry Barlow señala (1994: 8): «La información es una acción que ocupa tiempo en lugar de una presencia que ocupa espacio físico, como los artículos materiales». Según esta sentencia, utilizamos la información como un proceso no estático de flujo de conocimiento, donde el único elemento físico que interviene es el contenedor donde reside esa información. "Incluso cuando ha sido encapsulada en alguna forma estática como un libro o un disco duro, la información sigue siendo algo que nos ocurre cuando la descomprimimos mentalmente de su código de almacenamiento» (Barlow, 1994: 8). A esto habría que añadir ciertas particularidades económicas a los bienes informacionales que afectan a su función de demanda. El factor fundamental no se sitúa en su coste de producción, como sucede con la mayoría de los bienes materiales, sino en la demanda y los gustos de los consumidores. De hecho, los bienes informacionales tienen unos costes sustanciales en la producción de la copia original, pero prácticamente marginales en la (re)producción de las copias sucesivas (Shapiro y Varian, 1999: 4), lo que sitúa el precio real de la información en su «valor colectivamente atribuido", no en su coste de producción.

Podemos tratar la música, por ejemplo, como un buen ejemplo de bien informacional. La música grabada sólo ocupa tiempo y la única materia física que media es su envase, ya sea un disco de vinilo, un CD o un reproductor de MP3. De acuerdo con esto, la acción de escuchar música puede ser definida como el proceso que sucede cuando, al reproducir ese material sonoro, tenemos la capacidad de descodificar su información en una percepción estética. Además, la música, en tanto expresión artística audible, se puede caracterizar por su consumo recurrente. En otras palabras, las probabilidades de escuchar un buen disco de manera reiterada son mayores que las de volver a leer un buen libro, por ejemplo (Shapiro y Varian, 1999: 87). Hasta ahora, nuestra forma tradicional de consumo de música grabada - que no fuese emitida por un medio de comunicación de masas como la radio - se limitaba a la compra de un contenedor donde residía y se podía reproducir en cualquier momento 
(Chanan, 1995; Howland, Kenney y William, 1999; Kennedy y McNutt, 1999). Esta forma de consumo vino condicionada por los sucesivos avances en tecnologías de difusión, lo cual proporcionó un estatus económico a la expresión creativa objetivada, el álbum, que requería de una forma de retribución a sus creadores, el disco. El problema surge cuando, al proveer un estatus económico a esa información, tenemos que restringir su acceso sólo a aquellos individuos que han pagado por ella. Los creadores de expresiones estéticas (como músicos, cineastas o escritores) utilizan para ello los copyrights o derechos de autor. Consecuentemente, los músicos disfrutan de un mecanismo que les brinda la posibilidad de explotar comercialmente (Andersen y Frenz, 2010) un bien informacional, la música, encapsulado en un formato físico, el disco. Andersen y Frenz (2010) analizan de esta manera el conflicto entre retribución y evolución tecnológica: cuando aparecen evoluciones en las tecnologías de grabación o reproducción del material — por ejemplo: cintas magnéticas, CDs o archivos compresores de audios como el MP3 - y en las tecnologías de difusión — radio, televisión o Internet—, se abre la posibilidad de realizar «la copia masiva no autorizada» de dicho material (2010: 716), con lo cual se salta el medio de retribución económica que tenían los creadores.

Parte de lo que hace que Internet sea tan innovador - incluso revolucionario- en el campo de la comunicación es la posibilidad de consumir música, así como otros bienes informacionales, sin que medie ningún tipo de contenedor físico, lo cual hace más palpable su naturaleza fluida. En otras palabras, y tal y como afirmaba Barlow (1994), el intercambio de información a través de la red se puede metaforizar como un espacio donde se intercambia «vino» (información) sin su contenedor habitual, la «botella» (el soporte físico), en un proceso de reproductibilidad del objeto que tiende a ser infinito.

En el contexto actual de la sociedad de la información, el individuo no es un mero receptor de información sin capacidad de interactuar con el mensaje - caso de la radio o la televisión-, sino que se hace partícipe del propio intercambio, eligiendo, consumiendo pero también creando, modificando y emitiendo (Díaz et al., 2009). Este grado de interactividad e intercomunicación permite a los individuos compartir contenidos que ya poseen, sin privarse de su disponibilidad, como así ocurre con las propiedades físicas (Barlow, 1994; Horrigan y Reina, 2002; Rodman y Vanderdonckt, 2006). Este nuevo escenario de expansión reticular de la información está provocando que las tecnologías de la comunicación ligadas al intercambio estén siendo concebidas como una seria amenaza por aquellas industrias ligadas a la creación, la producción y la distribución de propiedad intelectual (Rojek, 2005). El no poder controlar directamente el acceso a esos bienes a través de la limitación física de un bien codificado y encapsulado, como ocurría con el disco, o a través del pago por un bien informacional concreto, como las tiendas virtuales tipo Amazon o iTunes, supone una de las amenazas más reiteradamente señaladas por la industria y los creadores. Para ahondar en este aspecto, presentaremos a continuación una de las tecnologías más comunes en Internet que permiten realizar el intercambio libre de bienes y contenidos informacionales, y que ha 
sido objeto de discusión entre la comunidad académica sobre su papel en el decline de la industria cultural: las redes peer-to-peer o P2P.

\section{Las redes peer-to-peer}

Los protocolos P2P definen «un tipo de redes electrónicas de comunicación en las cuales todos los participantes son servidores de almacenamiento de archivos, de forma que ofrecen servicio de descarga de dichos archivos al resto de miembros de la red» (Sanduli y Martín-Barbero, 2005: 108). Estos autores afirman que las redes P2P han aparecido como una nueva forma de conducción de contenidos, lo cual ha fracturado la cadena de valor en los que se sustenta la industria cultural o de contenidos. Michel Bauwens define estas dinámicas de interacción humana de un modo más emancipatorio:

Las dinámicas peer-to-peer [entre iguales] son procesos sociales en los cuales quienes forman parte de una red distribuida pueden sumarse libre y voluntariamente a la búsqueda de objetivos comunes. Son procesos que están surgiendo en cada pliegue de la vida social, como un tercer modo de crear valor más allá del mercado y de lo público. Implican una revolución en lo que concierne a la producción, el autogobierno y la propiedad. (Bauwens, 2005: 2)

El debate actual sobre si es lícito o no -incluso moralmente aceptableeste tipo de protocolos radica en el modo de conciliar, por un lado, leyes que protegen la propiedad intelectual de los creadores artísticos y, por otro, el derecho de los usuarios a disfrutar de espacios donde puedan «expresar, celebrar y compartir formas de ocio» ${ }^{1}$ (Rojek, 2005: 363). Este convenio, que es en sí fácil de enunciar, es extraordinariamente complejo de acordar entre los distintos agentes que intervienen en el debate sobre el intercambio de bienes y contenidos culturales en la era digital. Se prestan dos posiciones fundamentales al respecto.

Por un lado, una buena parte de los creadores y de la industria defienden que el nuevo paradigma digital ha traído la ruina al sector cultural debido a una relación directa e inversamente proporcional entre un aumento de las descargas y una disminución de los ingresos por ventas. Dado que Internet puede ofrecer una manera gratuita de hacer disponible la música, así como tantos otros contenidos, los discursos contra el intercambio de archivos se han extendido. Internet es a menudo concebida como una "tecnología disruptiva» capaz de representar una amenaza a la propiedad intelectual y a las empresas (Horrigan y Reina, 2002). Se hace necesario, pues, desde esta perspectiva, establecer nuevas formas legales de sanción de comportamientos y preservación de los derechos de propiedad intelectual, aunque eso soslaye, de modo colateral, el derecho al libre intercambio sin ánimo de lucro entre individuos.

1. Este derecho se materializó en la Declaración de Sao Paulo (1998) en un acto organizado por la World Leisure and Recreation Association, junto con el Servicio Social Do Comercio y la Latin America Leisure and Recreation Association. 
Por otro lado, una parte de creadores, distribuidores y, sobre todo, usuarios defienden que, debido al innegable nuevo contexto de reproductibilidad de los bienes culturales en la era digital, debe crearse un nuevo e histórico «contrato social» entre agentes (Bauwens, 2005; Bauwens en Fernández-Savater, 2010). La crisis del sector debiera atajarse mediante la búsqueda de nuevos esquemas de generación de ingresos que permitan ganarse la vida a los creadores reproductores (Bauwens, 2005) y que permitan, a su vez, realizar el libre intercambio de contenidos entre individuos.

La incertidumbre y el nerviosismo que la era de la información ha impregnado en el sector de la cultura se hacen visibles en el modo en que se percibe el intercambio. Desde multitud de medios, agentes, representantes y discursos en general, se ha estigmatizado el comportamiento individual del intercambio. Como han señalado Rodman y Vanderdonckt (2006), muchos periodistas han descrito a las personas que comparten contenidos a través de Internet como "piratas», "expoliadores» y «ladrones», y sus artículos son, en gran parte, sobre la búsqueda de "soluciones» técnicas al "problema» del intercambio de archivos. Rodman y Vanderdonckt (2006) señalan quienes son, a su juicio, las verdaderas víctimas de esta situación:

[...] las víctimas más visibles del intercambio de archivos en estos discursos no son músicos ya -muchos de los cuales han adoptado estrategias para compartir archivos y/o han condenado a la industria por su negligencia sistemática de su hacer (Love, 2000)—, son los conglomerados multinacionales del espectáculo, cuyo futuro está supuestamente en peligro a menos que puedan encontrar la manera de transformar la actual situación en un intercambio de archivos rigurosamente vigilado, controlado colectivamente y de alta rentabilidad comercial. (2006: 246)

Por alusión, el argumento de la industria cultural es que, desde que aparecieron los software de intercambio de contenidos (Napster fue el primero en 2000), las ventas de música y de cine han sufrido un continuo descenso (Dugan, 2000). La Asociación de la Industria Discográfica de América (RIAA) argumenta que los programas $\mathrm{P} 2 \mathrm{P}$ son «una pieza relativamente sencilla de software que se puede mover desde el escritorio de un programador adolescente a [convertirse en] un fenómeno de Internet capaz de amenazar a toda una industria» (Miller y Huffstutter, 2000, escribiendo acerca de lo que supuso Napster). A pesar de que existen investigaciones que afirman que el descenso de ventas comenzó antes de que este tipo de software apareciera (Dugan, 2000; Shields, 2009), ya se ha realizado el cálculo o la estimación sobre las pérdidas ocasionadas al sector por estas tecnologías de intercambio de información.

La asociación de la industria discográfica comercial en Gran Bretaña, la British Phonographic Industry (BPI), calcula que la descarga ilegal de música por intercambio de archivos costó a la industria 200 millones de libras en 2009 (Shields, 2009). Un informe elaborado por la consultora independiente Tera Consultants, a iniciativa de la Cámara Internacional de Comercio (International Chamber of Commerce, BASCAP), calculó las pérdidas de ingresos por la venta 
de CDs en la industria europea de la música provocadas por la tasa de sustitución de material pirata. La venta de discos físicos había sufrido un descenso del 61\%. En concreto, el informe estimaba que la piratería digital en España (los P2P) había provocado una pérdida de 413 millones de euros a la industria de la música en el periodo 2004-2008 (Tera Consultants, 2010: 29). Eso sin contar el impacto de la venta directa de material pirata en formato físico (conocido como top manta), que calculaban como un daño notablemente menor, aproximadamente diez veces menos. Entre las consecuencias de esas pérdidas económicas, provocadas «fundamentalmente por la piratería digital», está la pérdida de 13.200 puestos de trabajo a lo largo de todo el sector cultural, únicamente en España (2010: 8).

No sólo las grandes asociaciones industriales se han sentido amenazadas por los nuevos fenómenos de intercambio en la red. Una gran parte de creadores, técnicos, productores, editores y trabajadores en general del sector de la cultura se han unido en multitud de asociaciones y manifiestos para luchar contra lo que consideran "parásitos del negocio» (CopeerRight Agency España y La Coalición de Creadores e Industrias de Contenido, 2009). En España, representantes del mundo laboral de la cultura, creadores y trabajadores, así como la patronal del sector, han aunado fuerzas para la consecución de un mismo objetivo común: el mantenimiento de la industria creativa o industria cultural. Para ello, han creado una plataforma de intereses compartidos que demanda nuevas normativas legales que regulen la "piratería» en Internet: La Coalición de Creadores e Industrias de Contenido ${ }^{2}$. Este grupo de presión pretende concienciar sobre el hecho que cualquier medio de intercambio, descarga o visualización digital no autorizada de contenidos culturales se ha convertido en una práctica condenable, ilegal e inmoral, puesto que es causa directa de las pérdidas, tanto económicas como de empleo (CopeerRight Agency España y La Coalición de Creadores e Industrias de Contenido, 2009).

A tenor de este clima, la importancia de investigar todas las caras de este fenómeno poliédrico se presenta como una tarea necesaria, en particular, en el campo que nos ocupa: las ciencias sociales. El presente trabajo camina en esa dirección. Pretende ofrecer información empírica relevante para contrastar los diferentes argumentos que explican la realidad social del fenómeno y que, por tanto, dan pie a soluciones contrapuestas a la cuestión.

\section{Hipótesis}

En los últimos años, el número de estudios empíricos sobre el efecto del intercambio de archivos en la venta de música ha crecido notablemente. Si hay algo que caracteriza al conjunto de esta producción científica es la falta de consenso en cuanto al efecto que tiene un comportamiento (descargas) sobre el otro (compras). En la tabla 1, hemos pretendido

2. En el año 2009, esta demanda en contra de las «descargas ilegales» fue materializada por cerca de 2.500 músicos y trabajadores de la industria en general en el manifiesto La música es cultura, la música es empleo. 


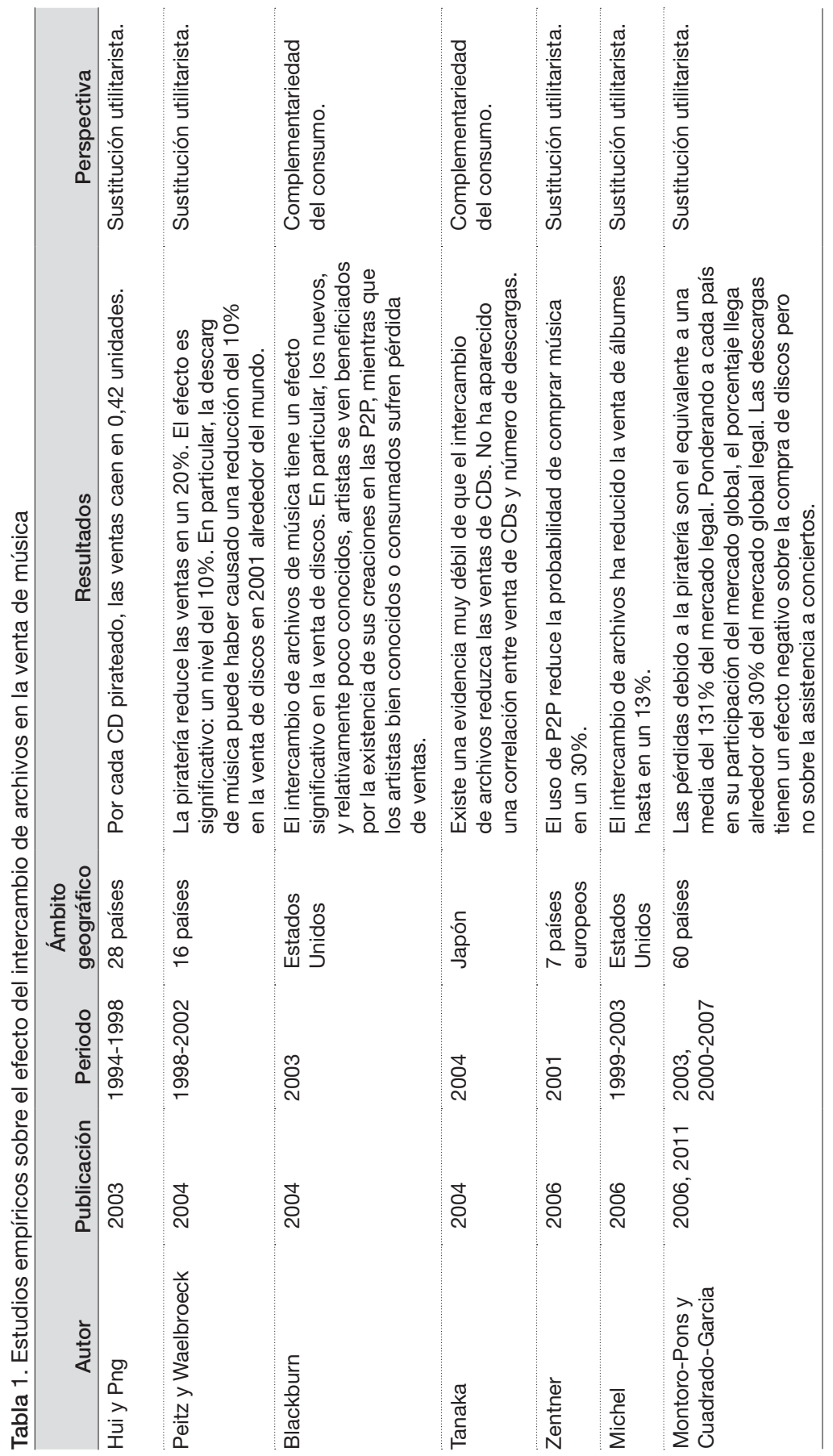




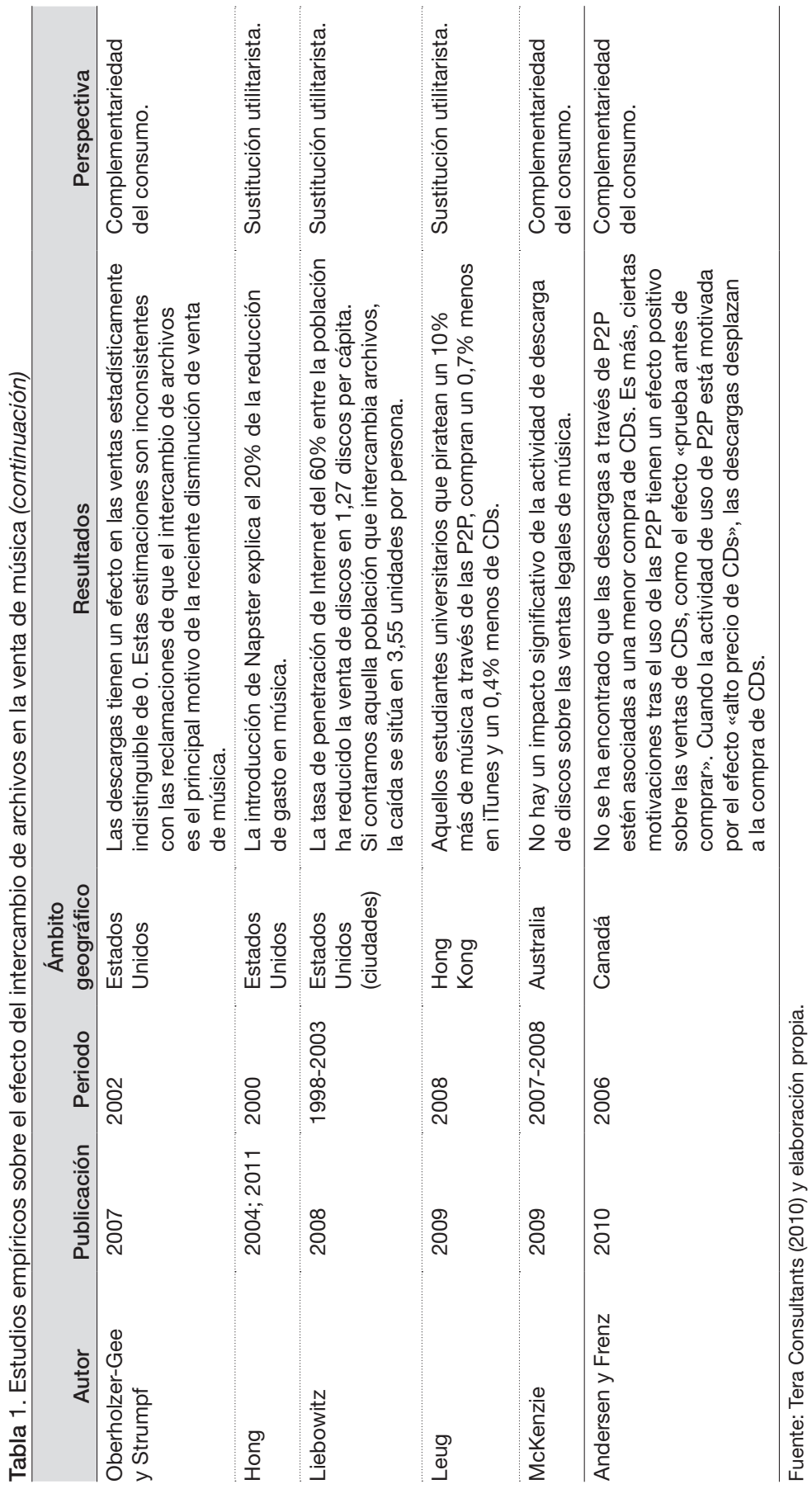


sintetizar algunos de los estudios más relevantes de la última década, colocados en orden creciente según el año de publicación y mostrando diferentes descriptores de cada uno: año de publicación, periodo de análisis, ámbito geográfico del estudio, resultados más relevantes y perspectiva en la cual puede ubicarse cada uno de ellos, a saber, la tesis de la sustitución utilitarista y la tesis de la complementariedad del consumo. Ambas tesis o perspectivas van a ser descritas con mayor detalle en el siguiente epígrafe, con lo cual se conforma nuestro marco de hipótesis a enfrentar en el análisis.

\section{H1. La perspectiva de sustitución utilitarista}

Un primer enunciado podría ser que aquellos individuos que más películas y más música se descargan son los que menos CDs originales compran. Es, en ese sentido, una sustitución de un bien que antes tenía un precio en el mercado y al que ahora se tiene acceso libre y gratuito. La motivación, por tanto, es puramente economicista.

H1A. Los individuos que menos compras realizan están sustituyendo la pauta de compra por la pauta de la descarga y/o copia digital

Según esta hipótesis, los individuos que más contenidos culturales se descargan están realizando un ejercicio de elección racional — desde una perspectiva puramente utilitarista-, puesto que están sustituyendo un comportamiento por otro (descarga por compra), con el objeto de maximizar su utilidad o su satisfacción individual (reducir costes económicos y ampliar su abanico de consumo) ${ }^{3}$. Por consiguiente, se deduce que aquellos individuos que seguirán manteniendo una pauta de consumo legal de contenidos culturales serán los que menos se descarguen.

\section{H1B. Los individuos que más compras hacen son los que menos descargas y/o copias realizan}

Su patrón coincidiría, según los argumentos de la perspectiva de sustitución utilitarista, con un comportamiento éticamente responsable, puesto que, a pesar de que disfrutan del acceso a esos bienes culturales a través de Internet,

3. Desde esta perspectiva utilitarista, se han realizado numerosos estudios sobre la motivación de los usuarios de las redes P2P para descargas, pero no tanto sobre el comportamiento de los que suben contenidos a estas redes para compartirlos (Nandi y Rochelandet, 2008). En este sentido, se ha conceptualizado la figura del free-rider de redes P2P (Adar y Huberman, 2000; Feldman et al., 2003; Asvanund et al., 2004), que se sirve de la red para descargar únicamente, frente a un perfil más «altruista» de usuario que no sólo descarga, sino que también colabora aportando contenidos (Nandi y Rochelandet, 2008). Debido a las limitaciones técnicas de la encuesta, nosotros no vamos a abordar el asunto de la subida de contenidos, que, a pesar de ser un tema crucial de los estudios sobre P2P, nos alejaría de nuestro objeto: el efecto de las descargas sobre la compra. 
prefieren seguir adquiriéndolos legalmente y en su formato tradicional, el físico ${ }^{4}$.

\section{H2. La perspectiva de la complementariedad}

Desde una perspectiva diferente, cabría pensar que los individuos que más contenidos se descarguen no tienen por qué estar haciendo una sustitución de un comportamiento por otro. Pueden estar simultaneando ambos patrones de consumo (compra y descarga).

H2A. Los individuos que más compras hacen son también los que más descargas ylo copias digitales realizan

La oferta y el acceso a contenidos en el mundo digital ha crecido exponencialmente y las posibilidades de acceso a cada uno de los contenidos excede la capacidad individual de adquirirlos — es decir, comprarlos individualmente a precio de mercado, tal y como vienen señalando Andersen y Frenz (2010). A los individuos que no suelen comprar contenidos culturales a través de CDs o casetes tampoco les ha afectado la llegada de las nuevas tecnologías. Su pobre perfil de consumidor cultural también se refleja en su patrón de consumo digital a través de redes $\mathrm{P} 2 \mathrm{P}$, puesto que son los que menos descargas realizan.

H2B. Los individuos que menos compras hacen son también los que menos descargas y/o copias digitales realizan

En otras palabras, los individuos tienen, en el nuevo contexto de reproductibilidad digital de la información, la capacidad y/o la oportunidad -incluso podríamos hablar del deseo y/o la necesidad — de consumir muchos más contenidos de los que son capaces de comprar a precio de mercado. Ahora bien, este nuevo contexto de interacción e intercambio de información no será más que un reflejo de los patrones y los hábitos de consumo cultural que cada individuo tiene en el mundo real sin tener en cuenta la red.

\section{H3. La participación cultural «ex-domus»}

La cuestión que emerge de la hipótesis H2 no es baladí. ¿Cómo se explica, entonces, que se haya producido una caída de ventas e ingresos de las industrias culturales en los últimos años, mientras que, al mismo tiempo, han aumentado las descargas? La respuesta no es sencilla, ni este estudio pretende contestarla en su totalidad. Aun así, presentaremos algunas pistas de carácter académico que contribuyan al conocimiento en la materia.

4. Presuponemos, cuestión que contemplamos en nuestro modelo de análisis ceteris paribus, que, tanto uno como otro perfil de individuos tienen acceso a Internet y que, por tanto, disfrutan de la posibilidad de acceso. Ahora bien, el acceso a Internet no garantiza que todos los individuos tengan conocimientos de los procedimientos a seguir para tener acceso a redes $\mathrm{P} 2 \mathrm{P}$. 
Los individuos podrían estar manteniendo - a un menor ritmo que antaño- su patrón de consumo de contenidos en formato físico (CDs o casetes) y, a su vez, estar derivando parte de su presupuesto cultural al consumo de contenidos en vivo (como, por ejemplo, los conciertos de música). En otras palabras, han decidido -intencionadamente o no - retribuir a los creadores de un modo más directo y, a su vez, disfrutar de una experiencia que cumple con sus expectativas de cálculo coste-beneficio, como son los conciertos de música en vivo.

H3A. Los individuos que acuden a más conciertos son también los individuos que más música adquieren legalmente mediante compra y que más música descargan y/o intercambian

Actualmente, la balanza entre el poder adquisitivo de los individuos y el total de contenidos culturales a los que tienen acceso a través de Internet está enormemente desequilibrada. Su decisión, pues, ha sido la de establecer un menor ritmo de consumo legal en formato grabado - y de ahí las perdidas en el sector- por un mayor consumo en formato digital, compartiendo y descargando, además de apostar por experiencias ex-domus, es decir, fuera de la vivienda, como los conciertos. Esta reorientación del consumo parece tener su evidencia en los datos recogidos por el Ministerio de Cultura ${ }^{5}$.

Por consiguiente, podemos desarrollar un modelo de análisis empírico a través de la explotación estadística de una encuesta, con lo cual pondremos en pugna las hipótesis $\mathrm{H} 1$ y H2. La hipótesis H3 propondría alguna explicación parcial al cambio de patrón de consumo de bienes y contenidos culturales, una vez confirmada la hipótesis H2. Nuestro objetivo no es delimitar las características sociales y de comportamiento que influyen en la descarga de música, sino encontrar aquellas que caracterizan la compra de discos y la asistencia a conciertos. Ambas actividades nos ofrecerán información de gran relevancia sobre el perfil del consumidor económico de música, sus características sociodemográficas y su relación con las prácticas digitales.

\section{Métodos y datos}

Utilizando datos de la Encuesta de hábitos y prácticas culturales de 2007 (Ministerio de Cultura y Fundación Autor-SGAE) ${ }^{6}$, vamos a realizar un modelo de regresión lineal y un modelo de regresión logística. La encuesta cuenta con una

5. En la última década, el número de conciertos en España ha pasado a ser de 72.276 celebrados en el año 2001 a 138.613 en el año 2008. El porcentaje de espectadores ha crecido en un 51\%, mientras que la recaudación ha aumentado en un $116 \%$, pasando de casi 85 millones de euros en 2001 a más de 183 millones en 2008 (Fundación Autor-SGAE y Ministerio de Cultura, 2009).

6. La utilización de los microdatos anonimizados de la encuesta está sujeta a las condiciones y al compromiso de utilización, firmados entre el Ministerio de Cultura y la Fundación Pública Andaluza Centro de Estudios Andaluces, en el marco del proyecto Estilos de vida culturales: Un análisis de los consumos y hábitos culturales en España, realizado en el año 2009. Adicionalmente, hay que destacar el riguroso trabajo de campo realizado en la encuesta con asesoramiento del Instituto Nacional de Estadística. 
Tabla 2. Datos estadísticos descriptivos. Frecuencias y puntuaciones medias de las variables utilizadas

\begin{tabular}{|c|c|c|c|c|}
\hline Variables categóricas & \multicolumn{2}{|c|}{ Frecuencias (\%) } & \multicolumn{2}{|c|}{$\mathrm{N}$} \\
\hline Género & \multicolumn{2}{|c|}{100} & \multicolumn{2}{|c|}{14.822} \\
\hline Hombre & \multicolumn{2}{|c|}{47,8} & \multicolumn{2}{|c|}{7.089} \\
\hline Mujer & \multicolumn{2}{|c|}{52,2} & \multicolumn{2}{|c|}{7.733} \\
\hline Estado civil & \multicolumn{2}{|c|}{100} & \multicolumn{2}{|c|}{14.241} \\
\hline Soltero o independiente & \multicolumn{2}{|c|}{37,0} & \multicolumn{2}{|c|}{5.271} \\
\hline Casado o en pareja sin hijos & \multicolumn{2}{|c|}{10,9} & \multicolumn{2}{|c|}{1.551} \\
\hline Casado o en pareja con hijos menores & \multicolumn{2}{|c|}{24,2} & \multicolumn{2}{|c|}{3.451} \\
\hline Casado o en pareja con hijos mayores & \multicolumn{2}{|c|}{27,9} & \multicolumn{2}{|c|}{3.968} \\
\hline Nivel educativo & \multicolumn{2}{|c|}{100} & \multicolumn{2}{|c|}{14.822} \\
\hline Menos de 8 años escuela & \multicolumn{2}{|c|}{15,7} & \multicolumn{2}{|c|}{2.327} \\
\hline Más de 8 años o bachiller, EGB o ESO & \multicolumn{2}{|c|}{44,7} & \multicolumn{2}{|c|}{6.622} \\
\hline Bachiller superior, FPI o FPII & \multicolumn{2}{|c|}{24,3} & \multicolumn{2}{|c|}{3.601} \\
\hline Diplomado, licenciado, doctor & \multicolumn{2}{|c|}{15,3} & \multicolumn{2}{|c|}{2.272} \\
\hline Situación profesional & \multicolumn{2}{|c|}{100} & \multicolumn{2}{|c|}{14.822} \\
\hline Trabajando & \multicolumn{2}{|c|}{49,9} & \multicolumn{2}{|c|}{7.393} \\
\hline Parado & & , 2 & & 14 \\
\hline Jubilado, incapacitado, otros & &, 4 & 2.8 & \\
\hline Estudiante & & 0 & 1.3 & \\
\hline Labores del hogar & & 5,6 & 2.3 & \\
\hline Otras variables independientes & & & & \\
\hline Posee dispositivo portátil MP3, Wav u otros & & 3,9 & 5.7 & \\
\hline Lee revistas culturales al menos $1 \mathrm{vez}$ al mes & & 5,4 & 2.2 & \\
\hline Variables asistencia a eventos culturales & & & & \\
\hline Haber ido a un concierto en el último año & & 1,6 & 4.6 & \\
\hline Variables continuas & Media & Desv. típ. & Mínimo & Máximo \\
\hline Edad & 48 & 19 & 16 & 102 \\
\hline $\mathrm{N}^{\circ}$ discos comprados último trimestre & 0,77 & 2,67 & 0 & 75 \\
\hline $\mathrm{N}^{\circ}$ discos grabados o descargados último trimestre & 1,38 & 6,93 & 0 & $100^{*}$ \\
\hline № horas lúdicas en Internet a la semana & 1,16 & 3,82 & 0 & 99 \\
\hline
\end{tabular}

Fuente: Encuesta de hábitos y prácticas culturales (2007) y elaboración propia.

${ }^{*}$ Respecto a esta variable de intercambio de discos, hemos decidido reducir los valores máximos para evitar outlayers que complicasen las representaciones gráficas. De este modo, hemos decidido asignar 100 discos grabados o descargados a aquellos individuos que afirmasen descargarse igual o más de 101 (14 cambios realizados).

gran cantidad de indicadores de consumo y prácticas culturales. Entre ellos, encontramos: número de discos comprados en formato físico, número de discos consumidos a través de la descarga en Internet (fundamentalmente, a través de programas $\mathrm{P} 2 \mathrm{P}$ ) y frecuencia de asistencia a conciertos de música en el último año, entre otros. La encuesta, realizada por muestreo bietápico y estratificado, cuenta con una muestra total de 16.000 individuos de 15 años en adelante residentes en España (muestra analítica $=14.241$ casos). En la tabla 2, se muestran los datos estadísticos descriptivos de las variables utilizadas en nuestro análisis. 
Algunas de estas variables sociodemográficas han sido recodificadas de las variables originales con el objeto de reducir las categorías y aumentar la parsimonia del modelo. Es el caso de las variables Estado civil, Nivel educativo y Situación profesional. La variable dependiente Haber ido a un concierto en el último año hace referencia a todos aquellos individuos que han ido a algún concierto, ya sea de música clásica o actual, al menos una vez en el último año. Como información de vital importancia para el presente trabajo, deberíamos añadir que la inmensa mayoría de la población española ni compra $(78,1 \%)$, ni descarga discos $(85,8 \%)$. No obstante, los análisis cuentan con una base numérica de unidades muestrales suficiente para la realización de los análisis de regresión. El número de individuos que afirma haber comprado al menos un disco en el último trimestre es de 3.251, mientras que el número de individuos que afirma haber descargado o intercambiado al menos un disco en el último trimestre es de 2.111. Además, tanto la compra como el intercambio tienen una gran variabilidad en la frecuencia de su práctica, empero una gran concentración de los consumos menos intensos (entre 1 y 5 discos por trimestre).

\section{Resultados}

A continuación, vamos a presentar los resultados de nuestro análisis sobre la descarga de discos, así como de las probabilidades de acudir a conciertos según el conjunto de variables explicativas seleccionadas. La tabla 3 presenta los resultados del modelo de regresión lineal de mínimos cuadrados (OLS) con la variable Número de discos (formato CD o casete) que ha comprado en el último trimestre.

Con el Beta tipificado hemos obtenido un valor porcentual de cómo aumenta o disminuye, según sea el valor positivo o negativo, la compra de discos para cada una de las variables utilizadas, ceteris paribus. Por ejemplo, la categoría de la primera variable, Mujer, habría que relacionarla con su categoría de referencia, en este caso, Hombre. El coeficiente Beta tipificado toma el valor de $-0,057$. Esto quiere decir que, manteniendo el resto de variables constantes, la mujer compra un $5,7 \%$ menos de CDs o casetes que el hombre, y esta relación es estadísticamente significativa a un nivel $<0,01$. No obstante, podemos traducir esta relación porcentual, difícil de concebir cuando hablamos de unidades como los discos, a la elaboración de una tasa tipificada de compra para cada una de las variables. Esta tasa tiene una interpretación distinta según sea la variable categórica o numérica. Por ejemplo: por cada 19 discos que compra el hombre, la mujer compra uno menos, 18. En el caso de la edad, no tendría sentido afirmar que, por cada año que tenga el individuo, éste comprará un 5,3\% menos de discos. Una lectura alternativa, utilizando la tasa tipificada de compra, puede ser la siguiente: a medida que aumenta la edad, los individuos compran menos discos. Así, por cada tres discos que se compra una persona de 25 años, otra de 44 años (19 años más) se compraría dos y otra de 63 años, sólo uno. Por otro lado, y atendiendo ahora al resto de variables sociodemográficas, los titulados univer- 
Tabla 3. Análisis de regresión lineal sobre el número de CDs o casetes comprados en el último trimestre

\begin{tabular}{|c|c|c|c|c|}
\hline & Coeficiente & Error Std. & $\begin{array}{c}\text { Beta } \\
\text { tipificado }\end{array}$ & $\begin{array}{c}\text { Tasa tipificada } \\
\text { de compra }\end{array}$ \\
\hline Mujer a & $-0,303^{\star *}$ & $(0,048)$ & $-0,057$ & -18 \\
\hline Edad & $-0,007^{\star \star}$ & $(0,002)$ & $-0,053$ & -19 \\
\hline Más de 8 años, bachiller, EGB o ESO b & 0,062 & $(0,071)$ & 0,011 & \\
\hline Bachiller superior, FPI o FPII & 0,113 & $(0,084)$ & 0,018 & \\
\hline Diplomado, licenciado, doctor & $0,305^{\star *}$ & $(0,092)$ & 0,041 & 24 \\
\hline Parado $^{c}$ & $-0,167$ & $(0,093)$ & 0,015 & \\
\hline Jubilado, incapacitado, otros & $-0,199^{*}$ & $(0,082)$ & $-0,029$ & 34 \\
\hline Estudiante & $-0,404^{\star *}$ & $(0,092)$ & $-0,044$ & -23 \\
\hline Labores del hogar & 0,008 & $(0,079)$ & 0,001 & \\
\hline Casado o en pareja sin hijos ${ }^{d}$ & $0,243^{\star \star}$ & $(0,078)$ & 0,028 & 36 \\
\hline Casado o en pareja con hijos menores & $0,181^{\star *}$ & $(0,062)$ & 0,029 & 34 \\
\hline Casado o en pareja con hijos mayores & $-0,003$ & $(0,066)$ & $-0,000$ & \\
\hline Haber ido a un concierto último año & $0,557^{\star \star}$ & $(0,052)$ & 0,097 & 10 \\
\hline Posee un reproductor MP3 & $0,208^{* *}$ & $(0,052)$ & 0,038 & 26 \\
\hline Lee revistas culturales & $0,472^{* *}$ & $(0,063)$ & 0,064 & 16 \\
\hline Horas lúdicas en Internet & 0,003 & $(0,006)$ & 0,004 & \\
\hline $\mathrm{N}^{\circ}$ discos grabados o descargados & $0,017^{\star \star}$ & $(0,003)$ & 0,045 & 22 \\
\hline Constante & $0,828^{\star *}$ & $(0,128)$ & - & -18 \\
\hline
\end{tabular}

N 14.241

Test F 0,000

$R^{2}$ ajustado 0,048

Fuente: Encuesta de hábitos y prácticas culturales (2007) y elaboración propia.

* Valor $p<0,05$.

** Valor $p<0,01$.

a. «Hombre» como categoría de referencia.

b. «Menos de primaria» como categoría de referencia.

c. «Trabajando» como categoría de referencia.

d. "Soltero" como categoría de referencia.

sitarios compran un 4,1\% más de discos que las personas sin educación. Las personas que se encuentran estudiando compran un $4,4 \%$ menos de discos que los ocupados, mientras que los jubilados lo hacen un 2,9\% menos. Las personas que se encuentran casadas, ya sea con hijos menores o sin ellos, compran alrededor de un 3\% más de discos que las que se encuentran solteras manteniendo el resto de variables constantes.

Seguidamente, prestaremos atención a las variables sobre hábitos culturales y digitales que incluimos en el modelo. La asistencia a conciertos aparece como la característica que más influye en la compra de música. Los asistentes compran un 9,4\% más de discos que los no asistentes. Es decir, por cada 10 discos que compra un no asistente, el asistente compra 11 manteniendo el resto de variables constantes. Además, tener un reproductor MP3 es una característica que, en lugar de disminuir la compra de discos en formato físico, la 
aumenta en un 3,8\%. Ser una persona con inquietud cultural, con deseos de sentirse informada a través de revistas culturales, también aumenta la compra de discos en un 6,4\%. Finalmente, comprobamos cómo las personas que más discos se graban o se descargan de Internet compran un 4,5\% más de discos en formato físico. Por cada 18 discos que se copia o se descarga un individuo, éste se compra uno en formato físico. Esta progresión se muestra mejor en la siguiente gráfica.

Como se observa en la gráfica 1, la relación entre el número de discos descargados o intercambiados y el número de discos comprados está ampliamente descompensada. Sin embargo, la relación, 18 a 1 de media, es creciente y directamente proporcional, ceteris paribus.

En resumen, la hipótesis sobre la complementariedad del consumo en el caso de España parece encontrar aquí evidencias que la sostengan, mientras que su hipótesis nula, la sustitución utilitarista, parece tener menos validez. En otras palabras, las personas que más discos compran son también las que más descargas y/o copias digitales realizan, mientras que aquellas que menos discos compran también son las menos voraces en el medio digital.

A continuación, pasaremos a realizar el análisis de regresión logística o elección discreta sobre la probabilidad de haber ido a un concierto de música.

Gráfica 1. Representación gráfica del número de CDs comprados en comparación con el número de discos descargados y/o intercambiados

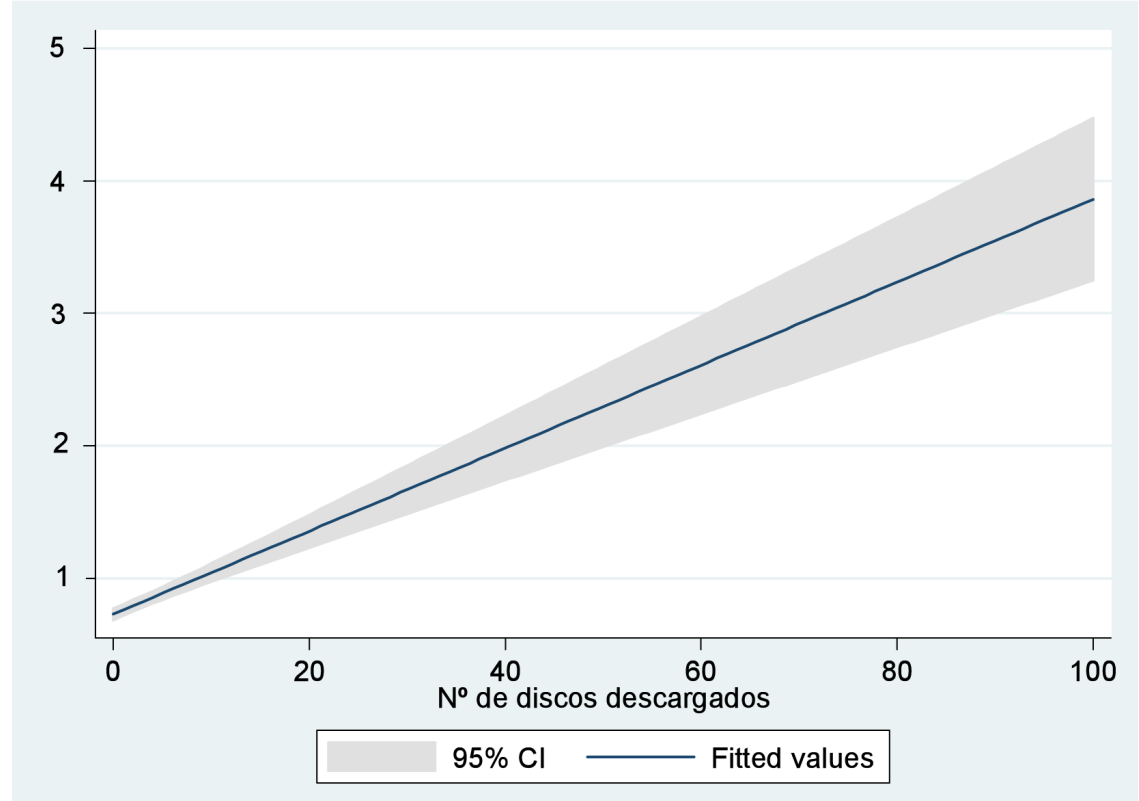

Fuente: Encuesta de hábitos y prácticas culturales (2007) y elaboración propia. 
Tabla 4. Análisis de regresión logística sobre la probabilidad de haber ido a un concierto

\begin{tabular}{|c|c|c|}
\hline & Coeficiente & Error Std. \\
\hline Mujer a & 0,076 & $(0,043)$ \\
\hline Edad & $-0,025^{\star \star}$ & $(0,002)$ \\
\hline Más de 8 años o bachiller, EGB o ESO ${ }^{b}$ & $0,681^{\star *}$ & $(0,092)$ \\
\hline Bachiller superior, FPI o FPII & $1,081^{* *}$ & $(0,098)$ \\
\hline Diplomado, licenciado, doctor & $1,417^{\star *}$ & $(0,102)$ \\
\hline Parado $^{c}$ & $-0,054$ & $(0,080)$ \\
\hline Jubilado, incapacitado, otros & $-0,206^{*}$ & $(0,084)$ \\
\hline Estudiante & $0,238^{\star *}$ & $(0,077)$ \\
\hline Labores del hogar & $-0,185^{*}$ & $(0,078)$ \\
\hline Casado o en pareja sin hijos ${ }^{d}$ & $-0,364^{\star \star}$ & $(0,073)$ \\
\hline Casado o en pareja con hijos menores & $-0,594^{\star *}$ & $(0,055)$ \\
\hline Casado o en pareja con hijos mayores & $-0,188^{\star \star}$ & $(0,068)$ \\
\hline $\mathrm{N}^{\circ}$ CDs comprados & $0,085^{\star *}$ & $(0,009)$ \\
\hline Posee reproductor MP3 & $0,456^{\star \star}$ & $(0,044)$ \\
\hline Lee revistas culturales & $0,643^{\star *}$ & $(0,052)$ \\
\hline $\mathrm{N}^{\circ}$ horas lúdicas en Internet & 0,005 & $(0,005)$ \\
\hline $\mathrm{N}^{\circ}$ discos grabados o descargados & $0,010^{\star *}$ & $(0,003)$ \\
\hline Constante & $0,613^{\star \star}$ & $(0,131)$ \\
\hline
\end{tabular}

N 14.241

Bondad del ajuste $-7.412,346$

Pseudo R $\mathrm{R}^{2} \quad 0,172$

Fuente: Encuesta de hábitos y prácticas culturales (2007) y elaboración propia.

* valor $p<0,05$.

${ }^{* *}$ valor $p<0,01$.

a. «Hombre» como categoría de referencia.

b. "Menos de primaria» como categoría de referencia.

c. «Trabajando» como categoría de referencia.

d. «Soltero» como categoría de referencia.

Como se observa en la tabla 4, asistir a conciertos de música es una experiencia común entre hombres y mujeres, puesto que no se observan diferencias estadísticamente significativas. En cambio, sí que encontramos diferencias en el resto de variables sociodemográficas. Podemos observar como, a mayor nivel educativo, la probabilidad de asistir a un concierto aumenta. Otra de las características que aumentan la probabilidad de asistir a un concierto es el hecho de estar en una situación profesional transitoria, como, por ejemplo, ser estudiante. Del mismo modo, no haber formado (aún) un núcleo familiar, es decir, estar soltero, también aumenta las probabilidades de acudir a un concierto. La asistencia a conciertos es una práctica predominantemente extendida entre las cohortes jóvenes. Conforme aumenta la edad, las probabilidades de asistir a un concierto disminuyen, y ser jubilado o dedicarse a las labores del hogar en lugar de estar trabajando también disminuyen dichas probabilidades. 
Atendiendo ahora a las variables sobre hábitos culturales y digitales que incluimos en el modelo, encontramos algunas conclusiones relevantes. Como ya vimos en el análisis anterior, debíamos de encontrar una cierta relación entre consumo de discos y asistencia a música en vivo. Así ha sido: cuantos más discos compran, comparten o descargan los individuos, mayores son las probabilidades de asistir a conciertos. También se observa que estar equipados con reproductores de MP3 aumenta las probabilidades de asistir a dichos eventos. En otras palabras, los equipamientos que permiten la reproducción de copias digitales de música, como los MP3, benefician a los autores por otras vías de retribución más directas, como son los conciertos.

Ahora bien, se nos antojaría necesario establecer un método sencillo de averiguar si el intercambio de archivos es una característica tan determinante sobre la asistencia a conciertos. Si fuese así, tendríamos aquí una evidencia empírica sobre cómo el intercambio de archivos - fundamentalmente a través de redes $\mathrm{P} 2 \mathrm{P}$ - es el elemento fundamental que ha hecho derivar el presupuesto de los individuos en compra de discos, a un mayor gasto en experiencias ex-domus, como los conciertos. Para ello, utilizaremos una gráfica de comparación de incrementos de probabilidad.

En la gráfica 2, se muestra como aumenta la probabilidad de asistir a un concierto conforme aumenta el número de discos descargados. La línea inferior representa el incremento de probabilidad de asistir a un concierto para un individuo medio. La línea superior representa el incremento de probabilidad para un joven (30 años) que se encuentra estudiando y ya disfruta de algún título universitario. Como observamos, las características sociodemográficas del "Joven universitario» son más determinantes que la descarga o el intercambio de discos a la hora de predecir la asistencia a conciertos. Esto es así porque las probabilidades de ir a un concierto para un individuo medio que haya descargado o compartido cien discos, son menores que las de un joven universitario que no se haya descargado ninguno. En otras palabras, la asistencia a conciertos está más influenciada por características como la edad, la situación profesional y el nivel educativo, que por la sola práctica de compartir y/o descargar discos.

\section{Conclusiones}

El nuevo contexto que ha traído la revolución tecnológica en general, e Internet en particular, ha cambiado, de manera innegable, el modo de consumir bienes y servicios culturales como la música. El debate, por tanto, no debe centrarse en si ha tenido un efecto o no, sino en la dirección de ese cambio, en cómo se ha traducido en nuevos hábitos de la ciudadanía. Basándonos en los resultados aquí obtenidos, podemos afirmar que las prácticas de intercambio de música, particularmente en el escenario digital, no tienen porqué representar una amenaza para la industria de la música. De hecho, podrían ofrecer nuevas oportunidades de negocio. 
Gráfica 2. Incrementos de la probabilidad de ir a un concierto según el número de discos compartidos y/o descargados

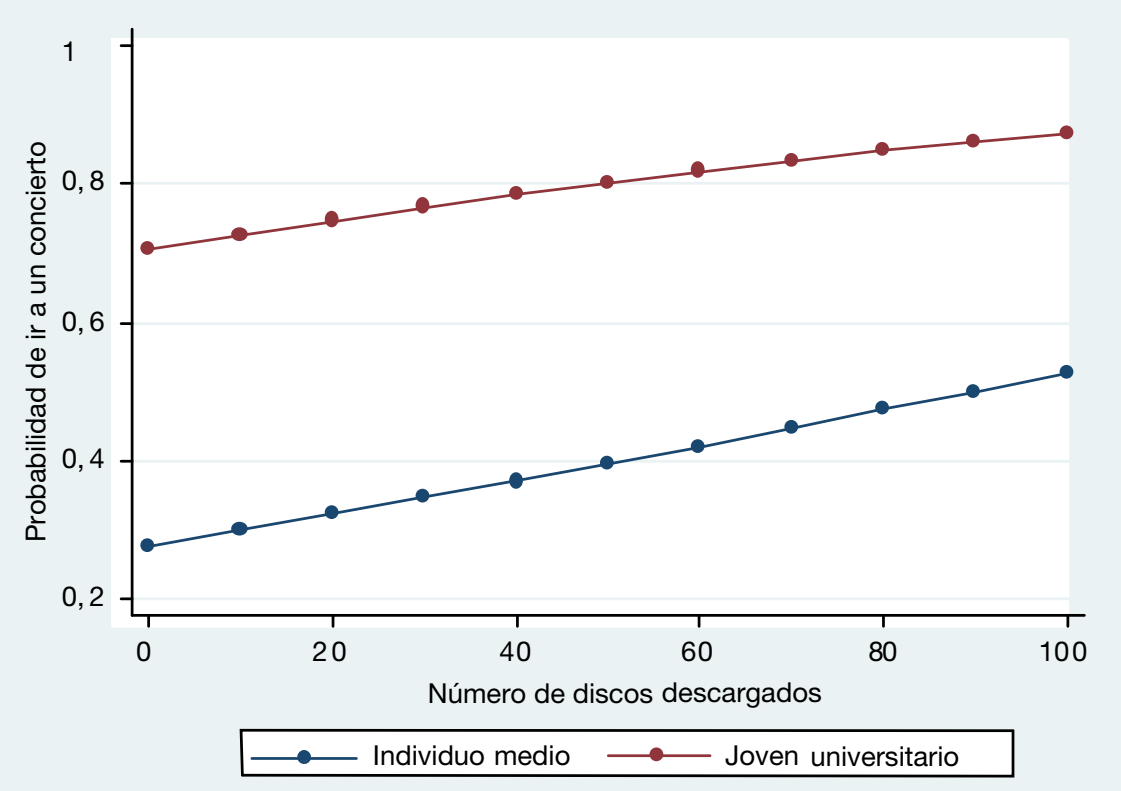

Fuente: Encuesta de hábitos y prácticas culturales (2007) y elaboración propia.

La utilización de redes de intercambio digital, como las P2P, representan un nuevo modo de desarrollar los comportamientos que ya estaban teniendo los individuos en el mundo offline. Dicho de otro modo, aquellos individuos que más descargas realizan son también aquellos que tienen un mayor consumo de discos físicos. Esto sugiere que dicha población está demostrando ya una cierta «disposición a pagar» (o willingness to pay) por los contenidos culturales que descarga y/o intercambia, lo cual resulta relevante a la hora de plantear nuevos modelos de retribución a los creadores.

El intercambio de música no puede ser concebido taxativamente como un comportamiento sustitutivo de la compra. Es más, en el presente trabajo hemos presentado evidencias que confirman cómo el intercambio es complementario a la compra y que, si bien no es la única característica, también influye positivamente en la asistencia a conciertos. Otras características sociales, como el nivel educativo, la situación socioprofesional o el ciclo vital asociado a la edad, a la paternidad o al estado civil, también influyen. En este sentido, nuestros análisis coinciden en parte con los resultados obtenidos por Andersen y Frenz (2010).

Las posibilidades actuales de acceso a la información hacen poco viable un modelo de retribución económica de los creadores, venta de discos mediante, 
como se daba antes de la llegada de Internet. De hecho, los individuos parecen estar derivando parte de su presupuesto, antes destinado fundamentalmente a la compra, hacia las experiencias en vivo, como los conciertos. El presente trabajo muestra igualmente indicios en esa dirección.

\section{Discusión}

Tras las conclusiones presentadas, hemos de tener en cuenta algunas reflexiones. El intercambio de bienes culturales, en tanto práctica de intensidades muy diferentes, va a tener efectos muy distintos sobre la compra de bienes culturales. Si bien hemos demostrado que los individuos que practican más intensamente el intercambio también son los compradores más voraces, su masa, en términos absolutos, es muy baja. Sólo un $5 \%$ de la población compra cinco o más discos por trimestre, proporción similar a la que sólo compran uno $(4,8 \%)$ y notablemente inferior de los que no compran ninguno $(78,1 \%)$. El temor de las posiciones conservadoras hacia el intercambio de archivos se fundamenta en aquellos comportamientos menos intensos o voraces, pero más comunes y frecuentes entre la población. En otras palabras, la pluralidad en la intensidad del intercambio no permite aseverar, de manera concluyente, que dicha práctica, como tal, sea ni beneficiosa ni perjudicial para la industria. No obstante, el presente trabajo muestra evidencias de cómo los que más descargan y/o graban, también son los que más compran, a pesar de que su importancia, en términos de volumen poblacional, es baja.

Lógicamente, para poder aseverar de un modo mucho más contundente la relación positiva entre un mayor intercambio y una mayor compra de música —o, al menos, disposición a pagar por ella—, así como entre intercambio y asistencia a conciertos, se hace necesaria la monitorización de los comportamientos individuales a través de estudios longitudinales. Para ello, resulta conveniente el establecimiento de métodos de recogida de datos primarios estables, continuados y con suficiente representación muestral. Ése es el caso de la Encuesta de hábitos y prácticas culturales. Por otro lado, la aseveración de vínculos causales entre el aumento del número de descargas y la disminución de las ventas no puede basarse en la utilización de datos agregados. Ello ha llevado a conclusiones basadas en relaciones espurias que no contemplan otros elementos o factores que influyen en el fenómeno. La utilización de datos de nivel micro nos acercarán más a la realidad de los fenómenos analizados y nos ayudarán a formular, con el suficiente rigor, fiabilidad y validez, soluciones consensuadas por todos los agentes.

\section{Referencias bibliográficas}

Adar, E. y Huberman, B.A. (2000). «Freeriding on Gnutella». First Monday, 5. Andersen, B. y Frenz, M. (2010). «Don't blame the P2P file-sharers: The impact of free music downloads on the purchase of music CDs in Canada». Journal of Evolutionary Economy, 20, 715-740. 
Asvanund, A.; Clay, K.; Krishnan, R. y Smith, M. (2004), «An Empirical Analysis of Network Externalities in Peer-To-Peer Music Sharing Networks». Information Systems Research, 15 (2), 155-174.

BARLOw, J.P. (1994). The Economy of Ideas: Selling Wine without Bottles on the Global Net [en línea]. <http://homes.eff.org/-barlow/EconomyOfldeas.html> [Consulta: 2 marzo 1994].

Bauwens, M. (2005). Peer to peer and human evolution: On "the P2P relational dynamic» as the premise of the next civilizational stage [en línea]. <http://economia.unipv. $\mathrm{it} /$ novita/seminario/P2PandHumanEvolV2.pdf $>$.

Blackburn, D. (2004). On-line Piracy and Recorded Music Sales. Manuscrito no publicado. Universidad de Harvard.

Chanan, M. (1995). Repeated Takes: A Short History of Recording and its Effects on Music. Nueva York: Verso.

Coalición de Creadores e Industrias de Contenidos. Manifiesto Derechos para todos en Internet [en línea]. <http://www.sgae.es/recursos/documentacion/Manifiesto_Derechos_para_todos_en_Internet.pdf $>$.

Copeerright Agency España y La Coalición de Creadores e Industrias de Contenido (2009). Parasite Business [en línea]. <www.youtube.com/ watch? $=$ g8LsFldJWcg $>$.

Cuadrado-García, M. y Montoro-Pons, J. (2011). «Live and prerecorded popular music consumption». Journal of Cultural Economics, 35 (1), 19-48.

Díaz, R. et al. (coord.) (2009). Código Fuente: la remezcla. Sevilla: Zemos98 - Gestión Creativo Cultural.

Dugan, S.M. (2000). «Napster Sends a Message to Music Industry: "Your Customers Aren't Happy"». Info World, 102, mayo.

Feldman, M.; Lai, K.; Chuang, J. y Stoica, I. (2003). "Quantifying Discincentives in Peer-To-Peer Networks». Working Paper. U.C. Berkeley.

Fernández-Savater, A. (2010). «El mercado se beneficia de la creatividad social, pero sólo devuelve precariedad». Público [en línea]. <http://blogs.publico.es/fueradelugar/category/michel-bauwens?doing_wp_cron> [Consulta: 10 abril 2010].

Fundación Autor-SGAE y Ministerio de Cultura (2009). Anuario SGAE de las artes escénicas, musicales y audiovisuales 2007-2009. <http:/www.artenetsgae.com/ anuario/home.html>.

Hong, S.H. (2004). «The Effect of Napster on Recorded Music Sales: Evidence from the Consumer Expenditure Survey». SIEPR Discussion Paper, 03-18.

Hong, S.H. (2011). "Measuring the Effect of Napster on Recorded Music Sales: Diference-in-diferences Estimates under Compositional Changes». Working Paper, University of Illinois. [en línea] <https://netfiles.uiuc.edu/hyunhong/www/napster. pdf> [Consulta: 16 julio 2012].

Horrigan, J.B. y Reina-Schement, J. (2002). «Dancing with Napster: Predictable Consumer Behavior in the New Digital Economy». IT\&Society, 1 (2), 142-160.

Howland, J.; Kenney, C. y William, R. (1999). Recorded Music in American Life: The Phonograph and Popular Memory, 1890-1945. Nueva York: Oxford.

Hui, K-L. y PNG, P. L. (2003). "Piracy and the Legitimate Demand for Recorded Music». Journal of Economic Analysis \& Policy, 0 (1), 11.

Kennedy, R. y Mcnutt, R. (1999). Little Labels - Big Sound: Small Record Companies and the Rise of American Music. Bloomington, Indiana: University Press.

Leug, T.C. (2009). «Should the Music Industry Sue Its Own Customers?: Impacts of Music Piracy and Policy Suggestions». Paper. Chinese University of Hong Kong. 
Liebowitz, S.J. (2008). «Testing File Sharing's Impact on Music Album Sales in Cities». Management Science, 54 (4), 852-859.

Love, C. (2000). «Courtney Love does the math». Salon.com [en línea]. <http://dir. salon.com/tech/feature/2000/06/14/love/index.html> [Consulta: 14 junio 2000].

Mckenzie, J. (2009). «Illegal music downloading and its impact on legitimate sales: australian empirical evidence». Australian Economics Papers, 48 (4), 296-307.

Miller, G. y Huffstutter, P.J. (2000). «File-Sharing PC Software Shakes Up Music World». The Los Angeles Times [en línea]. <http://articles.latimes.com/2000/ feb/24/news/mn-2069> [Consulta: 24 febrero 2000].

Montoro-Pons, J. y Cuadrado-García, M. (2006). "Digital goods and the effects of copying: An empirical study of the music market». International Conference on Cultural Economics. Viena, del 6 al 9 de julio.

Nandi, K. y Rochelandet, F. (2008). «The incentives for contributing digital contents over P2P networks: an empirical investigation». Review of Economic Research on Copyright Issues, vol. 5 (2), 19-35.

Oberholzer-Gee, F. y Strumpf, K. (2007). "The Effect of file Sharing on record Sales: An Empirical Analysis». Journal of Political Economy, 115 (1), 1-42.

Peitz, M. y Waelbroeck, P. (2004). "The Effect of Internet Piracy on Music Sales: Cross-section Evidence». Review of Economic Research on Copyright Issues, 1 (2), 71-79.

Rodman, G.B. y VAnderdonckT, C. (2006). «Music for nothing or, I want my MP3. The regulation and recirculation of affect». Cultural Studies, 20 (2-3), 245-261.

Rojek, C. (2005). «P2P leisure exchange: Net banditry and the policing of intellectual property». Leisure Studies, 24 (4), 357-369.

Sanduli, F. y Martín Barbero, S. (2005). "El usuario y el proveedor digital: el "reparto digital" en las redes P2P». Zer, 18, 105-121.

Shapiro, C. y Varian, H.R. (1999). The Information Rules: A Strategic Guide to the Network Economy. Harvard: Harvard Business School.

Shields, R. (2009). "Illegal downloaders "spend the most on music, says poll". The independent [en línea]. <http://www.independent.co.uk/news/uk/crime/illegaldownloaders-spend-the-most-on-music-says-poll-1812776.html> [Consulta: 1 noviembre 2009].

TANaka, T. (2004). «Does file sharing reduce music CD sales?: A case of Japan». IIR Working Paper. Hitotsubashi University Institute of Innovation Research, WP\#0508.

- (2011). «Do Illegal Copies of Movies Reduce the Revenue of Legal Products?: The case of TV animation in Japan». Discussion Papers series 11-J-010, Research Institute of Economy, Trade and Industry. Tokyo, Japan.

Tera Consultants (2010). Building a Digital Economy: The Importance of Saving Jobs in the EU's Creative Industries [en línea]. International Chamber of Commerce/ BASCAP [en línea]. <http://www.iccwbo.org/uploadedFiles/BASCAP/Pages/ Building\%20a\%20Digital\%20Economy\%20-\%20TERA(1).pdf>.

Wheaton, J.R.; Greene, D.A. y Cope, S.S. (2005). Amici curae brief of musical artists in MGM v. Grokster [en línea]. <http://www.eff.org/IP/P2P/MGM_v_ Grokster/20050301_artists.pdf>.

Zentner, A. (2006). "Measuring the Effect of File Sharing on Music Purchases». Journal of Law and Economics, 69, 63-90. 